\title{
Red herrings remain in geographical ecology: a reply to Hawkins et al. (2007)
}

\author{
Colin M. Beale, Jack J. Lennon, David A. Elston, Mark J. Brewer and \\ Jonathan M. Yearsley \\ C. M. Beale (c.beale@macaulay.ac.uk) and J. J. Lennon, The Macaulay Inst., Craigiebuckler, Aberdeen, AB 15 8QH, UK. \\ - D. A. Elston and M. J. Brewer, Biomathematics and Statistics Scotland, The Macaulay Inst., Craigiebuckler, Aberdeen, AB 15 \\ 8QH, UK. - J. M. Yearsley, Dept d'Écologie et Évolution, Univ. de Lausanne, CH-1015 Lausanne, Switzerland.
}

In a recent paper Hawkins et al. (2007) question whether the use of spatially explicit methods such as generalised least squares (GLS) is necessarily better than using simpler non-spatial methods such as ordinary least squares (OLS) for fitting linear models to spatial data. This is consistent with a wider review of the literature which found that $>80 \%$ of ecological publications analysing spatial data ignore spatiallyexplicit modelling methods (Dormann 2007). Since the autocorrelations of the error term may be arbitrarily close to zero, it cannot be argued that OLS is always substantially worse than GLS, as OLS is the special case of GLS when the correlations are set to zero. However, this should never be used as an excuse to ignore GLS and instead to adopt OLS as the norm for spatial data: doing so may hinder sound inference in geographical ecology. It is the need to model correlation structures correctly that has motivated the substantial bodies of research in statistical methodologies summarised for spatially correlated data by Cressie (1993) and for temporally correlated data by Diggle et al. (1995) and Chatfield (2003). Ignoring spatial autocorrelation is to treat correlated observations as independent, and so is a form of pseudoreplication which has long been discredited in ecology (Hurlbert 1984).

In this short response we make three main points: firstly, Hawkins et al. have misunderstood the "redshift" as described by Lennon (2000); secondly, the sub-sampling method they use to reach their conclusions is inappropriate; and thirdly, improved modelling of the covariance structure of the error term allows better statistical inference to be made from spatial datasets.

\section{Point 1}

Hawkins et al. focus on the issue of whether spatial autocorrelation causes systematic shifts (bias) in regression coefficients fitted with non-spatial methods. This, however, is not the "red-shift" identified in Lennon (2000). Rather, the red-shift refers to the likely over-representation of covariates with stronger spatial autocorrelation when using model selection with non-spatial methods, an issue not addressed by Hawkins et al.

\section{Point 2}

The approach taken by Hawkins et al. is to generate parameter estimates that are supposedly unaffected by spatial autocorrelation. They take multiple subsamples from given data sets and compare the distributions of regression coefficients obtained from the subsamples with the estimates obtained from the full data set. Whilst this is potentially a useful approach for determining whether future data collection need be so intensive, as a method for determining the relative abilities of OLS and spatially explicit methods for estimating regression coefficients from the full data set it is flawed: the mean across repeated subsamples will always be close to the regression coefficients estimated 
(a)

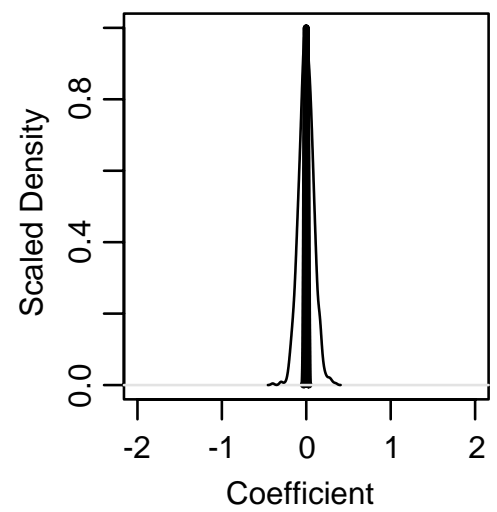

(b)

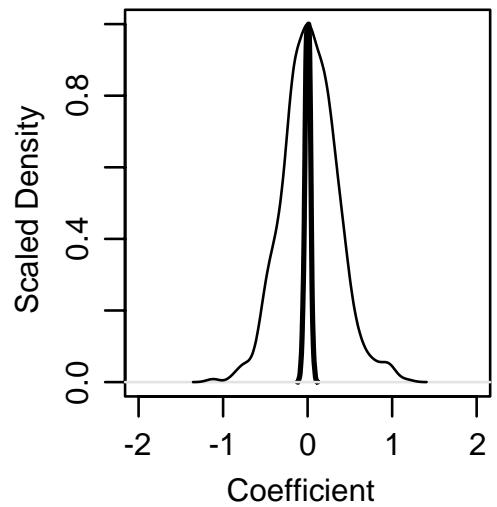

(c)

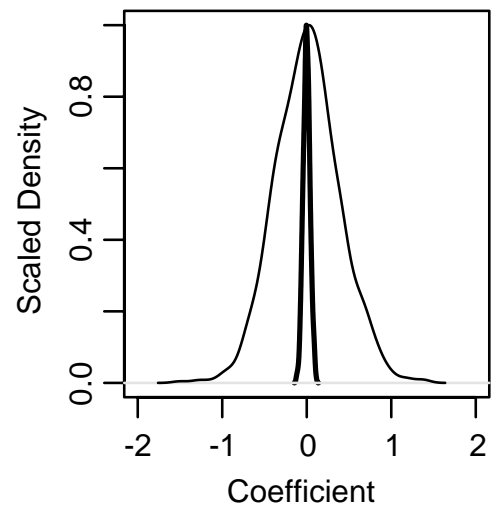

Fig. 1. Scaled density plot of estimated regression coefficients for 1000 simulations where the true coefficient is zero for all three covariates with increasing spatial autocorrelation from (a) to (c). GLS estimates are illustrated by the thick line and the thin line gives the OLS results. Y-values are scaled to ensure the maximum for both methods is one. Note that both methods are centred on zero but GLS estimates are far more precise, particularly as autocorrelation increases.

from the full dataset (as Hawkins et al. found, and as we also demonstrate by simulation in the appendices).

\section{Point 3}

The performance of methods for estimating model parameters is usually assessed in terms of bias (whether the estimate is too large or too small on average) and precision (how widely the estimates are spread). Both the OLS and GLS methods are always unbiased (this is demonstrated mathematically in Appendix 1) and so Hawkins et al.'s statements about bias are indisputably correct. Where the methods differ is in their precision in the presence of residual spatial autocorrelation, as demonstrated below.

To illustrate, we used 1000 simulations of spatiallystructured data sets. Methods and R code (R Development Core Team 2007) used in the simulations and analyses are provided as fully explained appendices: we invite readers to experiment for themselves. The true regression coefficients which we estimate from simulated data are always zero. We applied models using OLS, GLS and a version of Hawkins et al.'s subsampling method to each data set. The distributions of estimates of regression coefficients from OLS and GLS are provided in Fig. 1. These show clearly that the GLS estimates are much more precise than those (a)

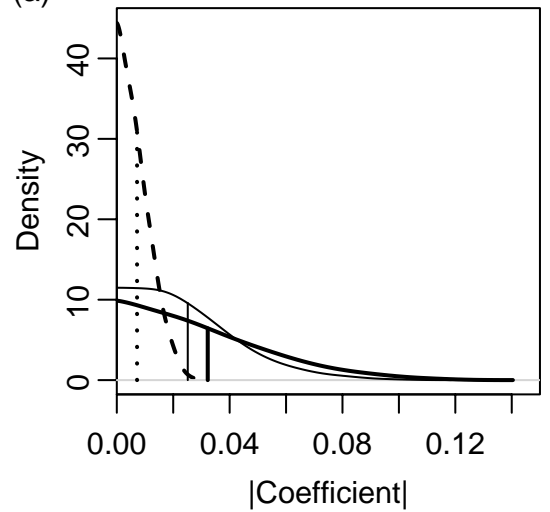

(b)

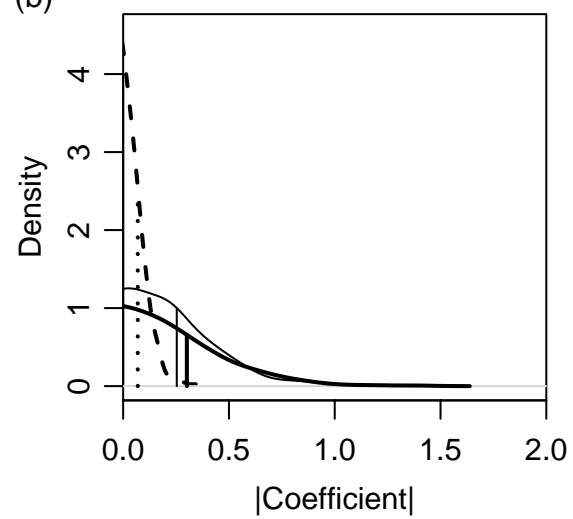

Fig. 2. Density plot of absolute values of regression coefficients estimated by (a) GLS and (b) OLS for 1000 simulations (see appendices for details). Note the difference of scales between (a) and (b), due to the more precise estimation by GLS. True parameter values underlying the simulations are 0 in all cases. The dashed, thin and thick lines represent estimates of parameters for covariates with low, intermediate and high autocorrelation, respectively. Mean absolute values of parameter estimates are identified by vertical lines. In all cases the mode is (correctly) 0 , but the mean absolute coefficient size gets progressively larger in magnitude with increasing spatial autocorrelation. 
from OLS. Alpargu and Dutilleul (2003) and others have used far more extensive simulations to explore different autocorrelation structures and relationships between variables, with the same general conclusions: for individual data sets, the OLS and GLS estimates may be very different, and that when residual autocorrelations are strong the OLS estimates are much less precise. Because of the low precision of the OLS estimates, it is also true that OLS estimates often have a greater absolute value than their GLS counterpart (Fig. 2). Both these results can be seen in Table 1 of Hawkins et al. (2007), in which each OLS estimate differs from its corresponding spatial estimate by more than one standard error and 21 of the 22 coefficients are larger in magnitude in the OLS models than their spatial counterparts. Similarly Dormann (2007) found that every coefficient in 24 separate ecological studies was larger in magnitude for OLS than spatially-explicit analyses of the same datasets.

\section{Conclusions}

We have demonstrated that the consequences of using OLS when GLS is more appropriate can be profound. If residual spatial autocorrelation is negligible or nonexistent, results from OLS and GLS should be equivalent, whilst if residual spatial autocorrelation is substantial it should be taken into account. Our experience is that spatially distributed response variables in ecological studies typically show spatial autocorrelation over and above that explained by the covariates. Consequently, the default analysis for spatial data should be to use methods which allow for spatial autocorrelation.

\section{References}

Alpargu, G. and Dutilleul, P. 2003. To be or not to be valid in testing the significance of the slope in simple quantitative linear models with autocorrelated errors. - J. Stat. Comput. Sim. 73: 165-180.

Chatfield, C. 2003. The analysis of time series: an introduction, 6th ed. - Chapman and Hall/CRC.

Cressie, N. A. C. 1993. Statistics for spatial data. - Wiley.

Diggle, P. J. et al. 1995. Analysis of longitudinal data. - Oxford Univ. Press.

Dormann, C. F. 2007. Effects of incorporating spatial autocorrelation into the analysis of species distribution data. - Global Ecol. Biogeogr. 16: 129-138.

Hawkins, B. A. et al. 2007. Red herrings revisited: spatial autocorrelation and parameter estimation in geographical ecology. - Ecography 30: 375-384.

Hurlbert, S. H. 1984. Pseudoreplication and the design of ecological experiments. - Ecol. Monogr. 54: 187-211.

Lennon, J. J. 2000. Red-shifts and red herrings in geographical ecology. - Ecography 23: 101-113.

R Development Core Team 2007. R: a language and environment for statistical computing. - R Foundation for Statistical Computing, Vienna, Austria, < http:// www.R-project.org >

Download the appendix as file E5338 from $<$ www.oikos.ekol.lu.se/appendix $>$. 